\title{
The End Point Tagger physics program at A2@MAMI
}

\author{
Oliver Steffen ${ }^{1, a}$, on behalf of the A2-Collaboration \\ ${ }^{1}$ Institut für Kernphysik, Johannes Gutenberg-Universität Mainz, Johann-Joachim-Becher-Weg 45, 55099 Mainz
}

\begin{abstract}
The A2-Collaboration uses a beam of real photons from the tagged photon facility at the electron accelerator MAMI in Mainz, Germany, to study photo-produced mesons. A new tagging device allows access to the higher photon beam energy range of 1.4 to $1.6 \mathrm{GeV}$. A large dataset containing more than 6 million $\eta^{\prime}$ and roughly 29 million $\omega$ decays has been obtained. Analyses are ongoing, including a study of the cusp effect and Dalitz plot in $\eta^{\prime} \rightarrow \eta \pi^{0} \pi^{0}$, giving insight to the $\pi \pi$ scattering length and the structure of the $\eta \pi \pi$ system, as well as the measurement of the electromagnetic transition form factor in $\eta^{\prime} \rightarrow e^{+} e^{-} \gamma$, a cross section measurement of $\gamma p \rightarrow 3 \pi^{0}$, and branching ratio analyses of $\eta^{\prime} \rightarrow \omega \gamma$ and $\omega \rightarrow \eta \gamma$.
\end{abstract}

\section{Introduction}

The A2-Collaboration uses the tagged photon facility at the Mainz Microtron MAMI [1] to study baryon resonances, meson photoproduction and meson decays.

After upgrading the existing experiment setup, a large dataset containing more than 6 million $\eta^{\prime}$ decays has been obtained during 10 weeks of beam time in 2014. It serves as a rich base for many physics topics to be studied. Analyses are ongoing, including the measurement of the electromagnetic transition form factor in $\eta^{\prime} \rightarrow e^{+} e^{-} \gamma$ is relevant for the understanding of $\eta / \eta^{\prime}$ mixing and theory of the anomalous magnetic moment of the muon (see section 3.1). A study of the cusp effect and Dalitz plot in $\eta^{\prime} \rightarrow \eta \pi^{0} \pi^{0}$, giving insight to the $\pi \pi$ scattering length and the structure of the $\eta \pi \pi$ system (see section 3.2). Branching ratio analyses of $\eta^{\prime} \rightarrow \omega \gamma$ and $\omega \rightarrow \eta \gamma$ will provide input for chiral effective theories (see section 3.3). The cross section measurement of $\gamma p \rightarrow 3 \pi^{0}$ will extend existing data from previous measurements (see section 3.4).

\section{Experiment}

The electron beam produced by the MAMI multistage racetack microtron has an energy $E_{\text {beam }}$ of up to $1.6 \mathrm{GeV}$ [1]. It is converted into bremstrahlung photons by a thin copper foil. The electron, together with the unused portion of the beam, is bent away by the tagging spectrometer magnet (see Fig. 1). The energy of the scattered electron $E_{\text {scattered }}$ is measured by a hodoscope, and the photon energy can be calculated: $E_{\gamma}=E_{\text {beam }}-E_{\text {scattered }}$. Forming a time coincidence

\footnotetext{
a e-mail: steffeo@uni-mainz.de
}

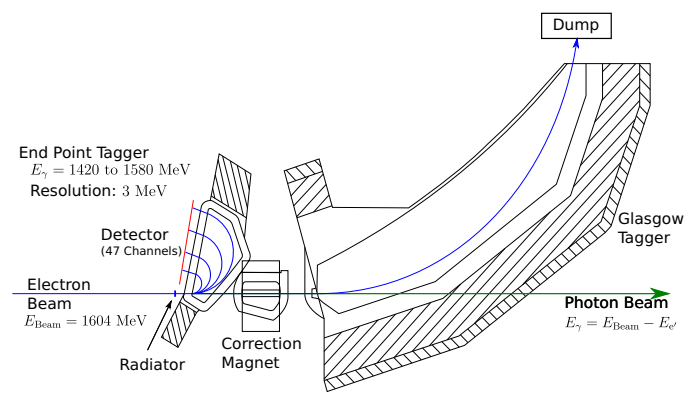

Figure 1. The photon tagging setup: Bremsstrahlung is created by shooting electrons on a thin metal foil. The energy of the scattered electrons is measured by a magnetic spectrometer. The specialized End Point Tagger is mounted in front of the main tagger, followed by a correction magnet.

with the detector system of the experiment makes it possible to energy tag the photons.

A new tagging device, the End Point Tagger, was constructed in 2014. It focuses on the highest photon energies of the bremsstrahlung spectrum from $1.4 \mathrm{GeV}$ to $1.6 \mathrm{GeV}$ with a resolution of $3 \mathrm{MeV}$. It is an ideal tool to study rare and comparatively heavy mesons like the $\eta^{\prime}$ and the $\omega$, produced in photo production reactions off the proton (see Fig. 2). Together with upgrades and fine-tuning of the readout system the existing experimental setup could be turned into an $\eta^{\prime}$ factory.

The photon beam impinges on a $10 \mathrm{~cm}$ long cell filled with liquid hydrogen, acting as a high density proton target. The target cell is contained in a cryostat and cooled down to temperatures below $20 \mathrm{~K}$. 


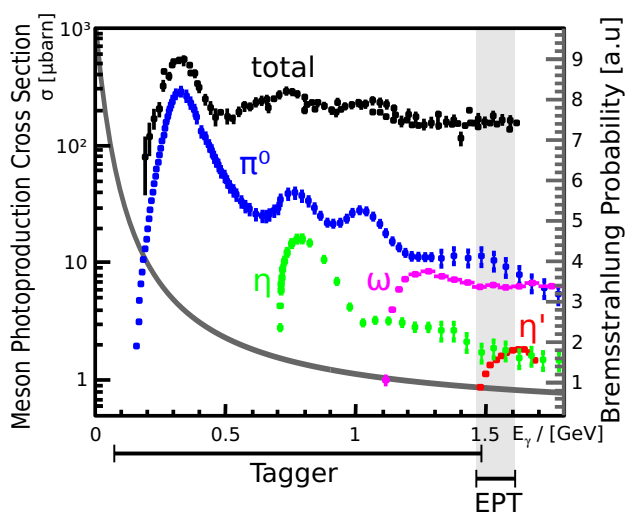

Figure 2. Sketch of photoproduction cross sections for prominent mesons (points) in the energy range covered by the Crystal Ball/TAPS experiment, together with the energy distribution of the bremsstrahlung spectrum (line). The End Point Tagger focuses on a narrow energy region around the $\eta^{\prime}$ threshold, while the main tagger covers a wide range of the lower energies. This allows rejection of the much more probable low energetic background events.

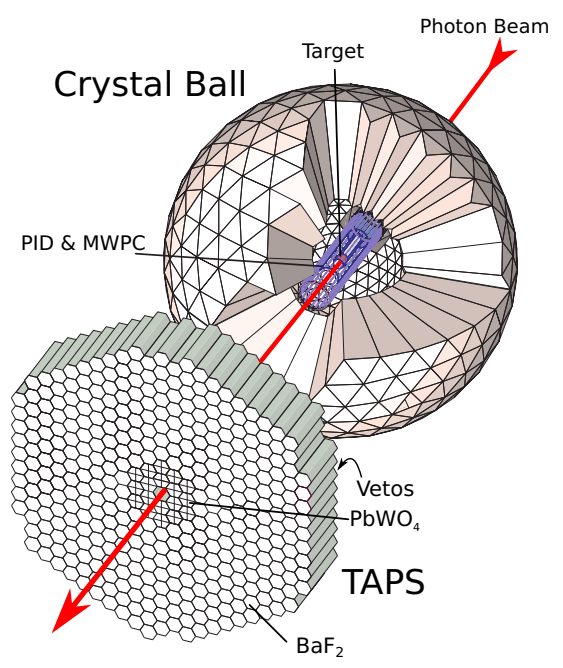

Figure 3. The detector system: The calorimeters Crystal Ball and TAPS cover over $96 \%$ of the solid angle. Additional particle identification detectors are located around the target and in front of TAPS.

The target is surrounded by the main calorimeter, the Crystal Ball [2]. It consists of 672 thallium doped sodium iodine crystals $(\mathrm{NaI}(\mathrm{Tl}))$, forming a sphere. The forward opening is covered by the TAPS detector [3], forming a wall of 366 barium fluoride $\left(\mathrm{BaF}_{2}\right)$ and additional 72 lead tungstate $\left(\mathrm{PbWO}_{4}\right)$ crystals. These detectors together cover over $96 \%$ of the full solid angle (see Fig. 3) and are ideal for the measurement of neutral final states. Both calorimeters are equipped with additional detectors made out of plastic scintillators, allowing particle identification by measuring the energy loss of traversing charged particles. Additionally, a pair of cylindrical multi-wire proportional chambers (MWPC) is located inside the Crystal Ball. Due to the extremely high rates they could not be used in the beam times mentioned above. Table 1 summarizes the properties of the detector system.

Table 1. Properties of the Crystal Ball/TAPS detector system.

\begin{tabular}{lcc} 
& $\mathrm{CB}$ & TAPS \\
\hline Energy Resolution & $\frac{2 \%}{\sqrt[4]{E_{\gamma} / \mathrm{GeV}}}$ & $\frac{0.8 \%}{\sqrt{E_{\gamma} / \mathrm{GeV}}}+1.8 \%$ \\
Crystals & 672 & 438 \\
Material & $\mathrm{NaI}(\mathrm{Tl})$ & $\mathrm{BaF}_{2}, \mathrm{PbWO}_{4}$
\end{tabular}

\section{Analyses}

\subsection{Electromagnetic Transition Form Factors}

The electromagnetic transition form factors (TFF) of pseudo-scalar mesons are important input for solving the mystery of the anomalous magnetic moment of the muon [4] and better understanding the $\eta / \eta^{\prime}$ mixing [5].

Currently a $3.6 \sigma$ discrepancy exists between then experimental observation [6] and theoretical predictions of $a_{\mu}=(g-2) / 2$. The theory is limited by QCD contributions $[7,8]$, consisting of hadronic vacuum polarizations and hadronic light-by-light scattering [9], where the transition form factors enter in the description primarily through the light-by-light scattering terms. The studies of meson decays allow the measurement of the $q^{2}$ dependent TFF in the timelike region $\left(q^{2}>0\right)$. They appear as a modification of the QED prediction of the differential cross section for the point-like meson:

$$
\frac{\mathrm{d} \sigma}{\mathrm{d} \Omega}=\left(\frac{\mathrm{d} \sigma}{\mathrm{d} \Omega}\right)_{\mathrm{QED}} \cdot\left|F\left(q^{2}\right)\right|^{2}
$$

Electromagnetic transition form factors of the $\pi^{0}[10]$ and $\eta[11]$ meson have already been studied in previous experiments of the A2-Collaboration (see Fig. 4). This experiment gives the opportunity of extending the existing data set to higher energies and also study the $\eta^{\prime}$ TFF in the $\eta^{\prime} \rightarrow e^{+} e^{-} \gamma$ decay, to complete the set of TFF of the light neutral mesons. The experiment covers a wide $q^{2}$ range in the time like region up to $q^{2} \approx 0.7 \mathrm{GeV}^{2}$. This allows a fit containing the $\rho$ peak region which is not covered by competing experiments [12]. A Monte Carlo simulation of the expected $q^{2}$ distribution is shown in Fig. 4. The analysis is still ongoing. 

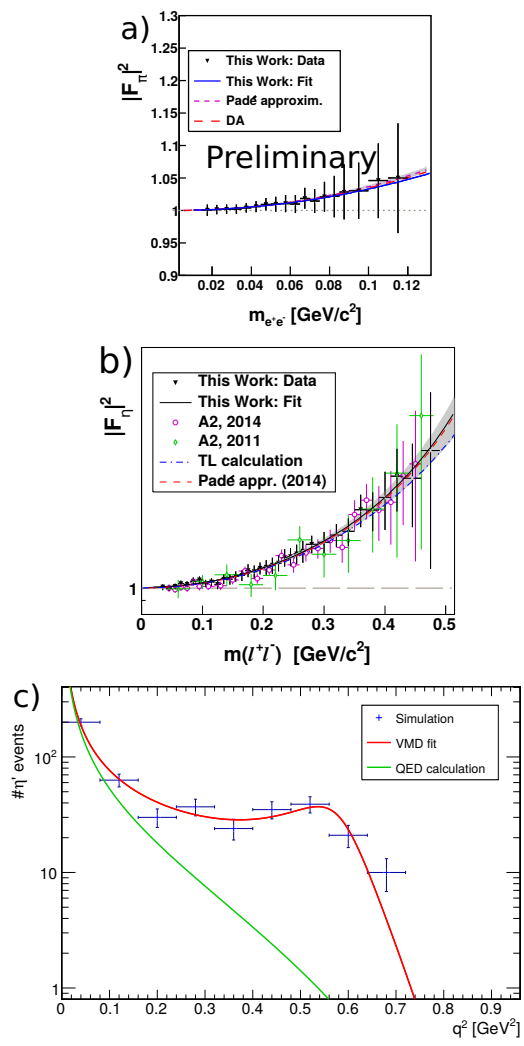

Figure 4. Electromagnetic transition form factors previously studied at A2: a) Preliminary $\pi^{0}$ TFF from $\pi^{0} \rightarrow$ $e^{+} e^{-} \gamma[10]$, b) $\eta$ TFF from $\eta \rightarrow e^{+} e^{-} \gamma[11]$, c) Monte Carlo simulation of the $q^{2}$ distribution of $\eta^{\prime} \rightarrow e^{+} e^{-} \gamma$, together with a VMD fit and the QED calculation.
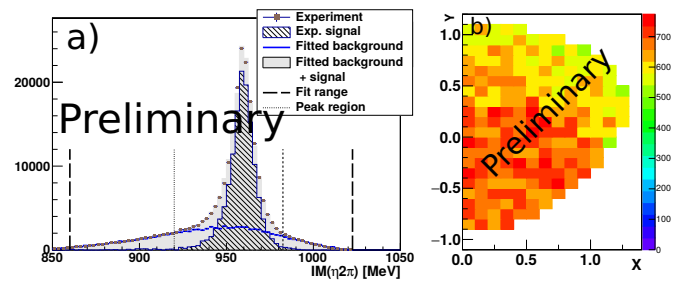

Figure 5. a) Invariant mass spectrum corresponding to one bin in the Dalitz plot. The peak from $\eta^{\prime} \rightarrow \eta \pi \pi$ is clearly visible above the background. b) One half of the symmetric Dalitz plot, containing $1.3 \times 10^{5}$ events.

\subsection{Cusp Effect and Dalitz Plot}

The large amount of $\eta^{\prime}$ allows detailed studies of the $\eta^{\prime} \rightarrow \eta \pi^{0} \pi^{0}$ decay. Two analyses of particular interest are the Dalitz plot analysis amd the search for the cusp effect.

A Dalitz plot analysis reveals the full information about the dynamics of a decay. The Dalitz plot variables $X$ and $Y$ for $\eta^{\prime} \rightarrow \eta \pi^{0} \pi^{0}$ can be calculated from the kinetic energies $T$ and masses $m$ of the decay products as follows:

$$
\begin{aligned}
& X=\frac{\sqrt{3}\left(T_{\pi_{1}}-T_{\pi_{2}}\right)}{Q} \\
& Y=\frac{\left(m_{\eta}+2 m_{\pi}\right)}{m_{\pi}} \frac{T_{\eta}}{Q}-1 \\
& Q=T_{\pi_{1}}+T_{\pi_{2}}+T_{\eta}=m_{\eta^{\prime}}-m_{\eta}-2 m_{\pi} .
\end{aligned}
$$

Since the two pions, $\pi_{1}$ and $\pi_{2}$, are indistinguishable both combinations have to be considered, making the plot symmetric in $X$. This Dalitz plot is well suited for testing extensions of chiral perturbation theory, such as large $N_{c}$ and resonance models [13, 14, 15]. The plot obtained during this experiment contains $1.3 \cdot 10^{5}$ events (see Fig. 5). This is a significant increase in statistics compared to previous experiments of the GAMS- $4 \pi\left(1.5 \cdot 10^{4} \eta^{\prime} \rightarrow \eta \pi^{0} \pi^{0}\right.$ events) [16] and BES-III (4.3 $10^{4} \eta^{\prime} \rightarrow \eta \pi^{+} \pi^{-}$events) [17] collaborations. The Dalitz plot parameters $a, b$, and $d$ can be extracted by fitting

$$
|A|^{2}=|N|^{2}\left[1+a Y+b Y^{2}+c X+d X^{2}\right]
$$

to the plot. The parameter $c$, describing the slope in $X$, cannot be extracted since the plot in this case is symmetric in $X$. The preliminary values from this analysis are compared to previous measurements and theory calculations in table 2 .

A cusp effect, due to $\pi^{+} \pi^{-} \rightarrow \pi^{0} \pi^{0}$ re-scattering, has been predicted for $\eta^{\prime} \rightarrow \eta \pi^{0} \pi^{0}$ [18] and is also being studied with the data set. This kind of effect was first observed in $K^{+} \rightarrow \pi^{0} \pi^{0} \pi^{+}$by NA48/2 [19], and has also been predicted for $\eta / K_{L}^{0} \rightarrow 3 \pi^{0}$ as a few percent effect. The analysis of the cusp would allow the determination of the S-wave $\pi^{0} \pi^{0}$ scattering length [20, 21].

\subsection{Branching Ratio Measurements}

Reactions between pseudo-scalar and vector mesons with a photon at the same vertex contain valuable input for implementing vector mesons in chiral effective field theories. The goal is a consistent picture of pseudo-scalar and vector mesons within the theory and finding a way to set up a power counting for unstable particles which to fulfill additional constraints due to spin 1 nature $[15,22]$.

The decay $\eta^{\prime} \rightarrow \omega \gamma$ has a branching ratio of $2.75 \pm 0.23 \%$ [23], and was measured by the BESIII collaboration as $\left(2.55 \pm 0.03_{\text {stat }} \pm 0.16_{\text {syst }}\right) \%$ [24]. A 
Table 2. Preliminary results for the Dalitz plot parameters in the decay $\eta^{\prime} \rightarrow \eta \pi^{0} \pi^{0}\left(\chi^{2} /\right.$ dof $\left.=233.6 / 195=1.198\right)$ with statistical errors, compared to the GAMS- $4 \pi$ measurement and predictions from large $N_{c}$ and resonance extensions of chiral perturbation theory.

\begin{tabular}{llll} 
Result & $\mathrm{a}$ & $\mathrm{b}$ & $\mathrm{d}$ \\
\hline A2 preliminary & $-0.073 \pm 0.078$ & $-0.072 \pm 0.013$ & $-0.054 \pm 0.009$ \\
GAMS- $4 \pi$ & $-0.067 \pm 0.016 \pm 0.004$ & $-0.064 \pm 0.029 \pm 0.005$ & $-0.067 \pm 0.020 \pm 0.003$ \\
Large $N_{c}$ extensions & $-0.098 \pm 0.048$ & $-0.050 \pm 0.001$ & $-0.092 \pm 0.008$ \\
Resonance ChPT & $-0.098 \pm 0.048$ & $-0.033 \pm 0.001$ & $-0.072 \pm 0.001$
\end{tabular}

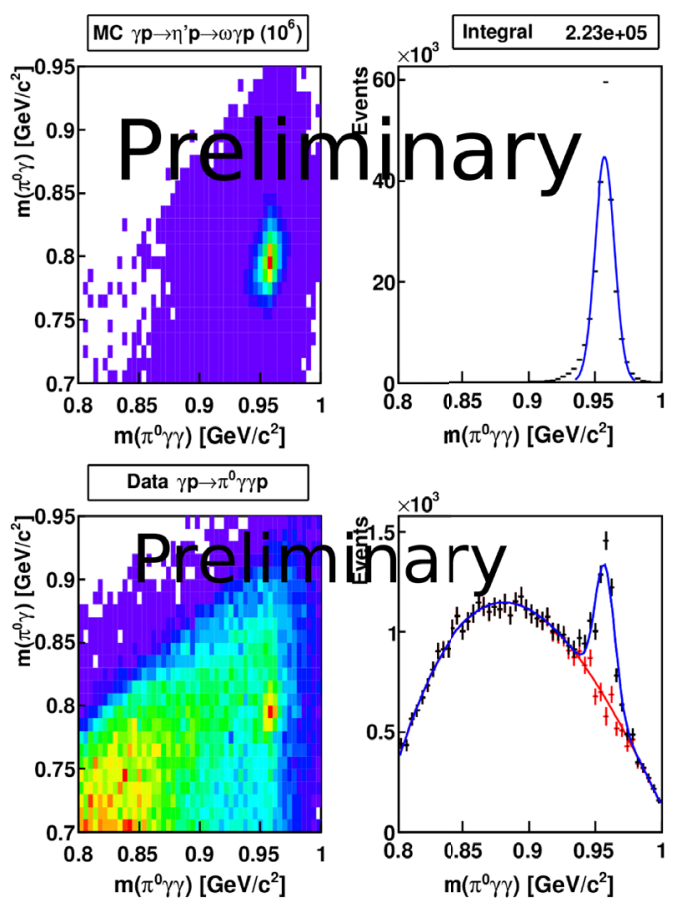

Figure 6. Branching ratio measurement of $\eta^{\prime} \rightarrow \omega \gamma$ : Preliminary invariant mass spectra from Monte Carlo (pure signal sample) and Data (including background).

branching ratio measurement of this decay relative to $\eta^{\prime} \rightarrow \gamma \gamma$ is being performed on our data set with about $10^{4}$ estimated signal events. Preliminary invariant mass spectra from Monte Carlo and experiment data are shown in Fig. 6.

Additionally about 29 million $\omega$ mesons were produced during this experiment, which allows the measurement of the $\omega \rightarrow \eta \gamma$ decay $\left(\Gamma_{i} / \Gamma=(4.6 \pm 0.4)\right.$. $\left.10^{-4}[23]\right)$. We are performing a relative measurement to $\omega \rightarrow \pi^{0} \gamma$, with an expected number of about 1500 signal events.

\subsection{Photoproduction cross section of $3 \pi^{0}$}

The photo production process of three neutral pions off the proton, $\gamma p \rightarrow p 3 \pi^{0}$, is a major background for the other ongoing analyses. This reaction is also

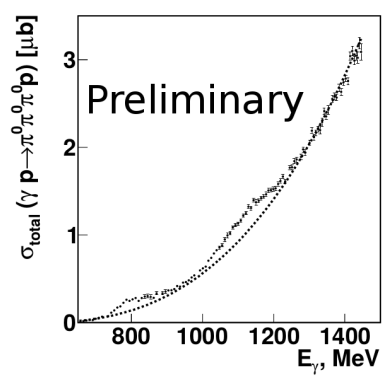

Figure 7. Preliminary result of a previous experiment measuring the three pion photoproduction cross section: $\gamma p \rightarrow p 3 \pi^{0}[25]$.

interesting to study as a production process. It can be described by the isobar model and involves a series of intermediate resonances. The cross section has been analyzed in previous experiments [25] (see fig 7). With the obtained data set we can extend and improve the previous results. Since the analysis is in an early state no preliminary results can be shown.

\section{Summary}

With the specialized tagging spectrometer and the upgraded detector readout of the Crystal Ball/TAPS experiment a large data set of $\eta^{\prime}$ and other mesons was recorded in 2014. Analyses are now ongoing within the A2-Collaboration: A study of the $3 \pi^{0}$ photoproduction cross section, a Dalitz plot analysis of $\eta^{\prime} \rightarrow \eta \pi \pi$, including the search for a cusp effect, as well as the measurement of the electromagnetic transition form factor in $\eta^{\prime} \rightarrow e^{+} e^{-} \gamma$, and branching fraction measurements of $\eta^{\prime} \rightarrow \omega \gamma$ and $\omega \rightarrow \eta \gamma$.

\section{References}

[1] M. Dehn et al., EPJ Special Topics 198, 19 (2011).

[2] M. J. Oreglia, PhD Thesis, Department of Physics of Stanford University (1980).

[3] R. Novotny, IEEE Trans. Nucl. Sci. 38, 379 (1991).

[4] E. Czerwinski et al., arXiv:1207.6556 [hep-ph], (2012). 
[5] L. Ametller, Phys. Scripta T99. 45 (2002).

[6] G. W. Bennett et al., Phys. Rev. D73, 072003 (2006).

[7] M. Davier et al., Eur. Phys. J. C 71, 1515 (2011). [Erratum: Eur. Phys. J.C 72, 1874 (2012)].

[8] K. Hagiwara et al. J. Phys. G 38, 085003 (2011).

[9] P. Masjuan, Nucl. Part. Phys. Proc. 260, 111 (2015).

[10] P. Adlarson et al., Phys. Rev. C 95, 025202 (2017).

[11] P. Adlarson, et al., Phys. Rev. C 95, 035208 (2017).

[12] M. Ablikim et al., Phys. Rev. D 92, 012001 (2015).

[13] R. Kaiser and H. Leutwyler, EPJ C 17, 623 (2000).

[14] R. Escribano et al., JHEP 2011, 94 (2011).
[15] G. Ecker et al., Phys. Let. B 223, 425 (1989)

[16] A. M. Blik et al., PAN 72, 231 (2009).

[17] M. Ablikim et al., Phys. Rev. D 83, 012003 (2011).

[18] Kubis, B., Schneider, S., EPJ C 62, 511 (2009).

[19] J.R. Batley et al., Phys. Let. B 633, 173 (2006).

[20] N. Cabibbo and G. Isidori, , JEHP 03, 21 (2005).

[21] C.-O. Gullström, A. Kupść and A. Rusetsky, , Phys. Rev. C 79, 028201 (2009).

[22] C. Terschlüsen, S. Leupold, and M. F. M. Lutz, Eur. Phys. J. A 48, 190 (2012).

[23] K. A. Olive et al., Chin. Phys. C38, 1 (2014).

[24] M. Ablikim et al., Phys. Rev. D 92, 051101 (2015).

[25] A. Starostin et al., arXiv:1101.3744 [nucl-ex], (2011) 